\title{
Représentation de la culture dans trois manuels
} de FLE

\section{Representation of Culture in Three FFL Textbooks}

\author{
Daniel ROJAS-PLATA ${ }^{1}$ (D)
}

${ }^{1}$ Prof. Dr., Universidad Autónoma Metropolitana, Department of Philosophy, Mexico City, Mexico

ORCID: D.R.P. 0000-0002-2545-3191

\section{Corresponding author: \\ Daniel ROJAS-PLATA, \\ Universidad Autónoma Metropolitana, \\ Department of Philosophy, \\ Mexico City, Mexico \\ E-mail: danielrojasplata@gmail.com}

Submitted: 14.02 .2020

Revision Requested: 10.03 .2020

Last Revision Received: 18.03.2020

Accepted: 20.03 .2020

Citation: Rojas-Plata, D. (2020) Representation of culture in three FFL textbooks. Litera, 30(1), 63-83. https://doi.org/10.26650/LITERA2020-0029

\section{RÉSUMÉ}

Dans ce travail, nous analysons les éléments culturels présents dans un ensemble de manuels de français langue étrangère. Nous nous concentrons principalement sur les éléments iconographiques, c'est-à-dire les photographies et les illustrations accompagnant chaque unité pédagogique. L'idée que la langue et la culture sont des produits interdépendants d'un seul processus est plus évidente dans le cas des manuels de langues, car ici la culture sert régulièrement de support pour représenter graphiquement la langue. Cette recherche a deux objectifs: 1) décrire l'emploi et l'évolution des images comme des éléments de représentation culturelle dans un ensemble de manuels de langue française et 2) analyser comment ces images se rapportent au contenu de différentes leçons. La méthodologie consiste à interroger trois manuels de français publiés à quelques années d'intervalle. Nous comparons quantitativement et qualitativement l'emploi des images dans ces matériaux et retraçons leurs caractéristiques et leurs différences. L'analyse révèle que dans les manuels les plus récents, il y a un plus grand nombre d'images, notamment de photographies. Celles-ci sont également plus complexes du point de vue de leur représentation culturelle. En général, les résultats soulignent un intérêt croissant à présenter un tableau multiculturel à travers l'emploi d'une iconographie très soignée dans les manuels de langues.

Mots-clés: Culture, langue française, iconographie, manuel de langues, analyse contrastive

\section{ABSTRACT}

In this paper, we analyze the cultural elements present in a set of French as a Foreign Language textbooks. We focus mainly on the iconographic elements, that is, the photographs and illustrations accompanying each unit. The idea that language and culture are interdependent products of a single process becomes even more evident in language textbooks, since here the latter regularly serves as a support to graphically represent the former. This research has two objectives: 1 ) to describe the use and evolution of images as an element of cultural representation in a set of French language textbooks and 2) to analyze how these images relate to the content of different lessons. The methodology consists of examining three textbooks of French as a Foreign Language that have been published a few years apart. We compare quantitatively and qualitatively the use of images in these materials and we retrace their characteristics and differences. The analysis reveals that in the most 
recent textbooks there is a greater number of images, especially photographs. These are also much more complex from the point of view of culture representation. In general, the results point to an increasing interest in presenting multiculturalism in language textbooks through the use of a well-defined iconographic collection.

Keywords: Culture, French language, iconography, language textbook, contrastive analysis

\section{EXTENDED ABSTRACT}

Nowadays, the language textbook is a frequent tool for teaching a foreign language. According to Ranchon (2016), in its realization, there is a convergence not only of linguistic and pedagogical questions, but also of commercial and cultural considerations. The idea advanced by some authors (see Ali et al., 2015) that language and culture are interdependent products of a single process becomes even more evident in language textbooks, since here the latter regularly serves as a support to graphically represent the former. Some authors (Auger, 2001; Chi, 2007) have analyzed the cultural representations that students draw from their contact with French. Although the corpus of these researches is a set of French textbooks, the central point here is the students' perception and not necessarily the differences between the cultural elements contained in the materials. So, the question is: Which culture is represented when teaching a language as widespread as French?

In this study, we analyze the cultural elements in the images of three French as a Foreign Language textbooks. The objective is to describe the use and evolution of images as elements of cultural representation and to analyze how these images relate to the content of different lessons. This theoretical and methodological approach is not accidental. A single image can contain a lot of symbols and iconographic expressions that are representative of a specific culture, so its appearance in a language textbook is certainly intentional. We limit the research to a particular group of textbooks which are quite well known in Mexico. The reason for this is to limit the scope of the corpus to a set of materials that have been used in a specific area.

The organization of the study is as follows. In the first part, we discuss the concepts of culture and representation from the point of view of current trends in language acquisition. We also describe the characteristics of the language textbook and the way in which culture is represented in them.

In the second part, we present the methodology which concerns the interrogation of three French as a Foreign Language textbooks: Le nouveauTaxi! (2009), Alter Ego Plus 
(2012) and Edito (2016). These are materials published a few years apart by two different publishers. The first two are currently in use in higher education institutions in Mexico and are a common tool for French courses. The third textbook is however not widely used in this context, but its marketing is recent (2016). We compare quantitatively and qualitatively the use of images in these materials and we retrace their characteristics and differences. We also establish what kind of images we will consider for the development of the analysis and propose a typology for their classification.

In the third section, we expose the results of the analysis. To keep an order in the presentation, we report the results of each textbook separately. We start with the quantitative results and continue with the qualitative results. We frequently make comparisons between textbooks and highlight the most relevant differences. The results indicate that in the most recent textbooks, there is a great number of iconographic elements belonging to different cultures. In these materials, we note a regular presence of personalities and institutions of the "Francophonie", a more detailed description of the countries where French is spoken and, in general, a larger number of images showing multiculturalism. In the same way, unlike the first textbooks where Francophonie has an illustrative function, the last textbooks present a more elaborate iconographic research work. Given these results, we can point out that there is an increasing interest in presenting multiculturalism in language textbooks through the use of a well-defined iconographic collection. 


\section{Introduction}

À l'heure actuelle, les manuels destinés à l'enseignement du français langue étrangère (FLE) constituent un outil fréquent dans la salle de classe. La quantité de manuels mis à disposition par les maisons d'édition est considérable et, très souvent, la sélection d'un ouvrage concerne non seulement des questions de méthodologie, mais également des considérations commerciales, sociales et/ou culturelles. En effet, le manuel peut être envisagé à la fois comme un livre permettant de structurer les cours de langue étrangère (LE), et comme un certain regard sur la langue et la culture.

Dans ce travail, nous analysons les éléments culturels présents dans trois manuels utilisés actuellement dans des cours de FLE au Mexique. L'étude vise à repérer les Éléments Iconographiques de Représentation Culturelle (EIRC) de ces manuels et à les comparer de manière quantitative et qualitative dans les contextes où ils apparaissent. Dans la première partie de ce travail, nous discutons les concepts de culture et de représentation du point de vue des courants actuels en LE. Nous décrivons également les caractéristiques du manuel et précisons de manière générale la représentation culturelle associée à la langue française dans le contexte mexicain. Dans un deuxième temps, nous présentons la méthodologie d'étude. Elle concerne l'interrogation de trois manuels de FLE : Le nouveau Taxi !, Alter Ego Plus et Édito. Les deux premiers manuels sont actuellement utilisés dans des institutions d'enseignement supérieur au Mexique (notamment I'Universidad Nacional Autónoma de México et l'Universidad Autónoma Metropolitana) et constituent un outil commun pour les cours de FLE destinés à un public grand adolescent/adulte. Le troisième manuel n'est pourtant pas très utilisé dans ce contexte, mais sa commercialisation est récente (2016). La troisième section est consacrée aux résultats de l'analyse sur corpus. Nous décrivons les EIRC contenus dans chaque manuel et les comparons. Cette analyse peut constituer un point de départ pour de futures recherches sur les manuels disponibles dans les pays et sur la représentation de la culture que les apprenants de FLE y trouvent.

\section{État de l'art : Culture et représentation dans les manuels de LE}

Il est essentiel d'établir une première approche sur la représentation de la langue et la culture françaises dans les cours de FLE, car elle constitue le point de départ pour les représentations des trois manuels formant le corpus. Dans cette section, nous faisons un passage en revue des concepts de culture et de représentation culturelle, ainsi que leur expression, dans les manuels de langue. 


\section{Concept de culture dans l'enseignement des langues}

De nos jours, le terme culture couvre un large éventail de notions complexes qui peuvent être définies en fonction de diverses disciplines : la sociologie, l'anthropologie, la philosophie, etc. II peut concerner tant des créations artistiques d'une communauté que des expressions ou des manières de comprendre la vie. D'un point de vue didactique, Galisson (1991, p. 117) et Porcher (1995, pp. 66-67) établissent une distinction fondamentale entre deux types de culture. La première, appelée « culture savante », se compose des produits artistiques conçus dans une société: peintures, monuments, écrits, etc. La seconde, appelée « culture populaire », relève de l'héritage intangible d'une communauté et peut concerner des éléments de la vie quotidienne ainsi que du savoir dire, être ou vivre (par exemple, les conceptions de l'amour, la mort ou le temps). C'est ce second sens qui apparaît le plus souvent dans des contextes d'enseignement des langues. Pour Brito Meyer (2013, pp. 55-56), la culture linguistique permet de saisir des aspects de l'identité psychosociale d'un groupe (pensée, morale, éthique, communication, comportement, etc.).

Au moment où l'apprenant fait face à une $L 2$, différents processus linguistiques et cognitifs sont mis en œuvre. Il s'avère que, dans les cours de LE, les apprenants découvrent un système linguistique nouveau mais aussi des éléments culturels nouveaux. Ce contact entraîne différentes réponses chez les apprenants. D'après Ali et al. (2015), la communication et la culture sont liées dans la mesure où elles accordent des valeurs et des significations aux différents éléments de la vie. Ce système de valeurs culturellement déterminées n'est pas toujours évident pour certains apprenants.' Dans ces cas, une explication s'impose très souvent afin de signaler les différences entre la culture cible et la culture d'origine. Cette information produit certainement un effet chez l'apprenant qui peut, par la suite, remettre en question ses propres concepts culturels (peut-être pour la première fois). De nombreux travaux sur l'apprentissage d'une L2 s'accordent sur le fait que les représentations culturelles des apprenants sont modifiées après leur contact avec une langue et une culture différentes. D'après Agar (1994), l'apprenant s'aperçoit que la culture qu'il essaie de comprendre et d'associer aux compétences linguistiques de son interlangue est visiblement différente de la sienne, et qu'il y a même des éléments qui lui sont totalement incompréhensibles du point de vue de sa

1 Chiswick et Miller (2005) ont même proposé des échelles permettant de mesurer le degré de séparation entre les langues. Leur méthodologie est basée sur la perception exprimée par les apprenants sur la difficulté d'apprendre une L2 spécifique. Ainsi, pour un locuteur anglophone, il serait plus difficile d'apprendre le mandarin que l'espagnol, tandis que pour un locuteur du mandarin, l'espagnol serait plus difficile à apprendre que l'anglais. 
propre culture. Ces différences peuvent l'amener à modifier les représentations de sa propre culture. Block (2007, p. 864) souligne que le sens d'identité est déstabilisé lors du contact entre différentes cultures et que les apprenants font des efforts cognitifs pour retrouver l'équilibre. Éventuellement les représentations culturelles nouvelles et anciennes trouvent un point de stabilité. D'après Puren (2002, pp. 60-61), cette interaction conduit à l'apprenant à réfléchir sur la culture d'autrui et la sienne, et à tirer des conclusions visant à construire une pensée multiculturelle.

Parmi les processus d'appréhension de la culture cible, on peut citer notamment le stéréotype ${ }^{2}$ qui apparaît fréquemment dans les cours des langues. Ce mécanisme permet aux apprenants de s'approprier de certains éléments culturels qui peuvent leur sembler étranges. Suivant Moore (2005, p. 10), le stéréotype a pour fonction de réduire à une représentation essentielle les données provenant de la réalité afin que celles-ci puissent être reconnues plus facilement par les individus. Cette hypothèse est également adoptée par Porcher (1995, p. 64) qui note que le stéréotype est une représentation partielle de la réalité. Dans cette optique, le stéréotype constitue une représentation qui peut être proche de la réalité ou bien proche de la caricature. Il est important enfin de mentionner que les stéréotypes constituent une première connaissance de la culture cible et peuvent persister même à des stades avancés de maîtrise de la langue.

\section{Quelle culture dans les manuels de FLE ?}

Le manuel de LE est un objet qui condense dans quelques peu de pages de nombreux éléments culturels d'une langue. Des textes, des enregistrements et des images constituent une source importante de représentations culturelles mise à la disposition des apprenants. Il est, à ce stade, nécessaire de définir ce que nous allons considérer comme manuel, ainsi que la manière dont la culture y est représentée, particulièrement en ce qui concerne l'enseignement d'une LE.

\subsection{Définition et organisation du manuel en LE}

Un manuel se distingue d'autres outils destinés à l'apprentissage des langues (comme les supports audio-visuels, les chansons, etc.) par sa structure, sa présentation

2 Le concept de stéréotype exposé dans cette étude correspond au sens utilisé dans l'apprentissage d'une L2. Il concerne particulièrement les représentations socioculturelles de personnes, de lieux et d'autres éléments appartenant à une communauté (Nguyen, 2008, p. 199). II s'agit d'une version certainement réduite de la notion développée par Schapira (1999) sur les types de stéréotypes (de pensée, de langue, etc.). 
et ses objectifs. Suivant Richaudeau (1986, p. 51), le manuel est un livre imprimé dont le but est de contribuer au processus d'enseignement-apprentissage d'une langue. Le choix et la structure des thèmes sont établis en raison d'une progression des compétences pragmatiques et sociolinguistiques, et en raison du contenu linguistique qui s'adapte aux différentes étapes d'apprentissage suivies par l'apprenant. Berringer (1995, p. 19) élargit cette notion en précisant que le manuel ne concerne pas seulement le support imprimé mais également les documents d'accompagnement (cassettes, vidéos, etc.). Par ailleurs, Girardet $(1995$, p. 28) fait une distinction entre les manuels de type universaliste et les manuels ciblés. Pour cet auteur, les premiers proposent des situations générales (souvent tournées vers l'international) sur l'emploi de la langue, tandis que les seconds peuvent être adaptés à un marché local et proposer des contenus plus spécifiques. Dans le cas du FLE, González Izquierdo (2014, p. 147) souligne que les modèles adoptés à présent dans la plupart des manuels scolaires sont basés sur l'approche actionnelle. Ici, on encourage vivement la communication dans des situations de la vie quotidienne aussi bien de la France que des pays francophones.

\subsection{Représentation culturelle dans les manuels}

Pour Ranchon (2016, pp. 29-32), le manuel de LE est à la fois un objet et un concept. D'après cet auteur, le contenu du manuel est sélectionné en fonction de critères linguistiques et culturels. Langue et culture sont ici « les résultats d'une modélisation de la réalité » (Ranchon, 2016, p. 27). Il est fréquent dans les cours de FLE que la langue qu'on apprend correspond au français standard, c'est-à-dire celle dans laquelle tous les membres d'une communauté linguistique se reconnaissent (Klinkenberg, 1999, p. 38). Bien qu'il existe des modules dédiés à la francophonie, ce français standard est souvent associé à la culture de la France.

Différents travaux ont analysé les représentations culturelles des apprenants de FLE par rapport à la langue française. Nous pouvons citer l'étude d'Auger (2001) sur les représentations de la France, du français et des français dans 42 manuels de FLE, ou bien les travaux de Chi (2007) et de Vajta (2012) sur les représentations des apprenants taïwanais et suédois sur la culture française. Cependant, les résultats de ces études ne concernent que le contexte dans lequel celles-ci ont été effectuées. En effet, les représentations culturelles que l'on peut se faire d'une communauté ne sont pas universelles. Dans le cas du Mexique, il n'y a pas beaucoup de travaux qui examinent ces questions. Nous pouvons citer la thèse de Barbier (2013) qui examine 
la représentation de la francophonie dans le manuel Latitudes, ainsi que les travaux de Silva (2011) et de Castillo et Pérez (2016) sur les attitudes et les motivations des apprenants de FLE au Mexique. D'après ces auteurs, la langue française y est souvent représentée comme une langue moderne, tournée vers l'international et associée fréquemment aux voyages. Elle est aussi perçue comme un instrument de promotion personnelle et professionnelle.

Comme nous l'avons indiqué, cette représentation initiale peut être modifiée lors des cours de FLE. Il est important d'analyser la manière dont les manuels cherchent à modéliser le contact entre apprenant et langue, et l'une des plus importantes est à travers l'emploi de photographies, de dessins et de tout autre élément iconographique que, dans notre étude, nous appelons Élément Iconographique de Représentation Culturelle (EIRC).

\section{Méthodologie d'analyse}

Pour cette étude, nous avons eu recours à trois manuels de FLE assez connus au Mexique. Il s'agit de manuels appartenant à deux maisons d'édition différentes et publiés à quelques années d'intervalle. Ils sont imprimés en couleur, de type universaliste et destinés à un public grand adolescent/adulte. Nous ne prenons en compte que le premier volume correspondant au niveau A1 du Cadre Européen Commun de Référence pour les Langues (Conseil de l'Europe, 2001). De même, nous analysons uniquement le livre imprimé et non le matériel d'accompagnement (CD, DVD-ROM) ni les ressources en ligne. La raison de ce choix tient au fait que ces matériels périphériques ne sont pas forcément utilisés en classe, car ils nécessitent des ressources spécifiques pour leur utilisation (ordinateur, connexion internet, etc.). Ils ne font donc pas toujours partie des outils proposés dans les cours de FLE et, dans de nombreux cas, ils ne sont même pas consultés.

La sélection de ces trois manuels tient à leur usage dans des cours de FLE au sein des principales institutions universitaires au Mexique (Universidad Nacional Autónoma de México, Universidad Autónoma Metropolitana et Instituto Politécnico Nacional), soit dans le cadre des cursus universitaires, soit dans des cours offerts au grand public. Nous décrivons ci-dessous les caractéristiques de chacun des manuels.

Le Nouveau Taxi! (Capelle \& Menand, 2009) [LNT] : Le plus ancien des manuels consultés. II comprend 144 pages imprimées en couleur, index et transcriptions 
des documents sonores compris. Il a été publié par Hachette en 2009 et est une version renouvelée de Taxi! publié en 2004. Ce manuel est actuellement utilisé par I'Universidad Autónoma Metropolitana Campus Iztapalapa dans ses cours de français et a été utilisé par les Alliances Françaises au Mexique il y a quelques années. Le livre est accompagné d'un DVD-ROM avec des exercices, des documents sonores et des vidéos complémentaires.

Alter Ego Plus (Kizirian et al., 2012) [AEP]. C'est un manuel largement connu dans des établissements d'enseignement nationaux et étrangers. Au Mexique, il est utilisé dans les centres de langues de l'Universidad Nacional Autónoma de México et dans diverses universités privées. Publié en 2012 par Hachette, il s'agit de la version renouvelée d'Alter Ego publié en 2006. Il est accompagné d'un DVD-ROM contenant des documents sonores, des dossiers et des portfolios. Il compte 223 pages, index et transcriptions compris.

Édito (Alcaraz et al., 2016) [EDI]. Publié en 2016 par Didier, c'est le plus récent des trois manuels. Il comprend 216 pages. Son usage dans des établissements d'enseignement du français n'est pas assez répandu au Mexique. À notre connaissance, il est seulement utilisé dans les niveaux avancés (B1 et B2) des cours de FLE dans les centres de langues de l'Universidad Nacional Autónoma de México. Son importance dans cette étude tient précisément à cette caractéristique : il permet d'établir une comparaison entre des manuels largement utilisés et des manuels moins connus dans des contextes d'apprentissage de FLE au Mexique.

\section{Classification des EIRC}

L'un des principaux éléments de représentation culturelle repéré dans les manuels de FLE est l'image imprimée. Une seule image peut contenir un bon nombre de symboles et d'expressions représentatives de la culture cible. Les images utilisées dans les manuels de FLE peuvent être classées en deux grandes catégories : photographies et illustrations.

Les photographies constituent une source importante de représentations culturelles, car elles concernent souvent des images de la vie quotidienne. Leur sélection dans un manuel peut être un indice du type de perspective méthodologique, linguistique et/ ou idéologique adoptée. Dans notre étude, nous les divisons en deux sous-catégories: 1) des images authentiques, en ce sens qu'elles cherchent à représenter des éléments réels de la culture cible (Figure 1), et 2 ) des images d'archives, qui peuvent être considérées 
peu représentatives du point de vue culturel (Figure 2). En effet, tandis que les images authentiques montrent des EIRC dans leur contexte, ce n'est pas le cas des images d'archives où l'on voit des situations génériques et dans des contextes neutres. Dans la Figure 1, les images de la ville de Pontoise peuvent aider l'apprenant à se situer culturellement. En revanche, dans la Figure 2, la caractérisation des individus n'est même pas nécessaire : une main, une tenue ou un profil peuvent suffire pour communiquer une certaine idée. Il est fondamental de souligner que, parfois, la classification d'une image dépend du co-texte qui l'accompagne. Par exemple, il peut arriver que l'apprenant ne reconnaisse pas immédiatement une façade apparaissant dans une photo, mais lorsqu'il est accompagné de la légende "Université de la Sorbonne », cet élément acquiert une valeur culturelle.

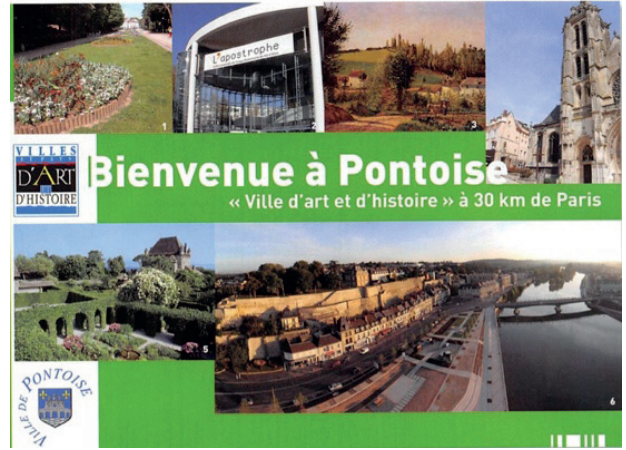

Figure 1: Image authentique (Source: $A E P$ )
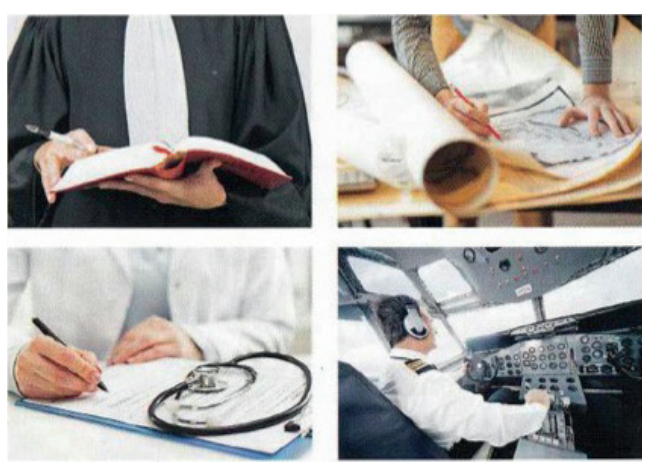

Figure 2: Image d'archives (Source: AEP)

Par ailleurs, les illustrations correspondent principalement à des dessins d'individus, d'objets ou de symboles. Nous distinguons deux sous-catégories : 1 ) illustrations authentiques (Figure 3) et 2) illustrations créées (Figure 4). La première catégorie correspond aux documents extraits de journaux, de bandes dessinées ou de magazines, aux dessins de monuments ou d'œuvres d'art, aux affiches, aux brochures, etc. Dans la Figure 3 , il y a une infographie sur le temps que les français consacrent aux tâches ménagères; ce document est obtenu d'une source réelle, ce qui fait penser qu'il est authentique. En revanche, les illustrations créées concernent des dessins génériques - par exemple des fruits, des vêtements, des meubles, etc. — qui ont été conçus pour expliquer ou exemplifier un thème, une activité ou un passage. Dans la Figure 4 , il y a des symboles renvoyant à différentes activités. Les illustrations sont très importantes pour notre analyse, surtout les authentiques, car elles concernent souvent des stéréotypes exprimant un élément culturel. Comme nous l'avons indiqué, certains 
stéréotypes peuvent être résumés en une caricature : physionomies, activités, gestes, vêtements, etc.

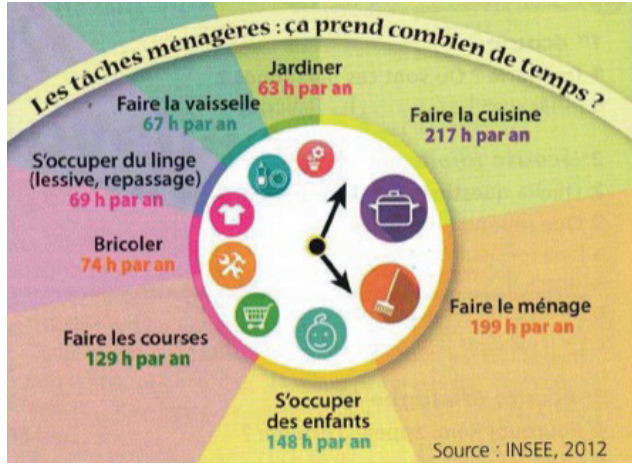

Figure 3: Illustration authentique(Source: EDI)

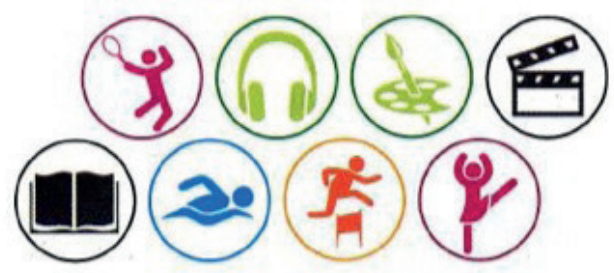

Figure 4: Illustration crée (Source: $A E P$ )

Enfin, pour l'étude nous ne prenons pas en considération les illustrations qui ne contiennent que du texte et qui sont génériques (e-mails, notes, listes, etc.), à l'exception de celles qui essaient de reproduire des éléments culturels authentiques : des affiches, des SMS, des publicités, des horaires de transports, etc.

\section{Interrogation du corpus et occurrences des EIRC}

Toute image qui répond aux critères établis dans la section précédente témoigne de l'occurrence d'un EIRC. Cependant, nous prenons comme une seule occurrence le groupe d'images appartenant à un même exercice. Par exemple, un ensemble de meubles est considéré comme une seule occurrence, au moins que chaque image ne soit différenciée par un numéro, car dans ce cas il s'agit d'éléments liés à des phrases qui s'opposent et qu'il faut distinguer. Cette décision tient au fait d'englober ces petites images qui sont souvent génériques et qui n'ajoutent pas d'informations pertinentes pour l'analyse culturelle.

Qualitativement, nous nous intéressons également au sujet traité dans l'unité pédagogique. Comme nous le verrons, il y a des thèmes qui favorisent l'apparition des EIRC dans les manuels. Ces unités pédagogiques sont particulièrement importantes du point de vue de la culture, car l'utilisation de stéréotypes est ici fréquente. En ce sens, il est fondamental d'analyser les variations existantes entre les différents manuels par rapport à ce sujet. 


\section{Résultats}

Dans ce qui suit, nous présentons les résultats de notre analyse sur les EIRC contenus dans les trois manuels proposés. Nous établissons une sous-section concernant chaque manuel afin de suivre un ordre dans l'exposition. D'abord, nous exposons les résultats quantitatifs et, ensuite, les résultats qualitatifs. Nous discutons également les données et proposons des comparaisons entre les différents manuels.

Il est important de mentionner que dans tous les manuels consultés, il y a au moins une section par unité consacrée à la culture/civilisation, ainsi qu'une section par manuel consacrée à la francophonie. Ces sections centrées sur la culture et le multiculturalisme sont fondamentales pour notre étude, car le recours au matériel iconographique y est fréquent, si bien que nous consacrons quelques lignes à la discussion détaillée de ces sections.

\section{Le Nouveau Taxi ! (LNT)}

Ce manuel se caractérise par une organisation très systématique des unités et des leçons. Chaque unité commence par la mise en œuvre d'une situation de communication. Au total, il y a 9 unités, elles-mêmes divisées en 4 leçons. La dernière leçon de chaque unité s'intitule «Arrêt sur » et correspond au composant culturel.

Le premier aspect iconographique à souligner est que chaque unité commence par une grande image accompagnant les objectifs généraux. À l'intérieur de chaque leçon, nous voyons également une image couvrant une cinquième partie de la feuille. En moyenne, il y a 1,3 images par feuille. Il est à noter que les sections de culture/civilisation de chaque unité contiennent davantage d'images que le reste des sections. Dans le Tableau 1, nous rapportons le nombre d'images par catégorie dans LNT (fréquence absolue et pourcentage).

Tableau 1: Nombre d'images retrouvées dans LNT

\begin{tabular}{|c|c|c|c|}
\hline \multicolumn{2}{|c|}{ Photographies } & \multicolumn{2}{c|}{ Illustrations } \\
\hline Authentiques & Adaptées & Authentiques & Créées \\
\hline $82(41 \%)$ & $40(20 \%)$ & $17(9 \%)$ & $61(30 \%)$ \\
\hline
\end{tabular}

D’après ces résultats, nous pouvons établir que les photographies authentiques et les illustrations créées sont les images les plus fréquentes avec un $41 \%$ et $30 \%$ de fréquence 
respectivement. En ce qui concerne les photographies, elles montrent des EIRC variés : monuments, symboles et paysages, principalement de la France. Nous trouvons également des expressions culturelles et des situations de la vie quotidienne française. Les individus apparaissant dans ces photos sont principalement blancs et parfois blonds.

Dans le cas des illustrations, elles deviennent de plus en plus sporadiques au fur et à mesure que les leçons progressent. Ainsi, pour les unités 7, 8 et 9, nous ne trouvons que deux illustrations. Les personnes sont dessinées de manière stylisée, au teint clair et aux traits fins.

En général, la quantité du texte est supérieure au nombre d'images par leçon. Par contre, dans la section de culture/civilisation, les images sont nombreuses. À partir du contexte où elles apparaissent, nous pouvons établir que la fonction des images est d'illustrer l'unité pédagogique ou l'exercice. Par exemple, dans l'unité des achats, la photo d'un magasin y apparaît ; pour les voyages, c'est le cas des guichets ; pour l'itinéraire, une carte ; et ainsi de suite.

En ce qui concerne les icônes culturelles, nous trouvons qu'elles sont principalement liées à la culture de la France : Édith Piaf, un marché de Noël et les feux d'artifice sur I'Arc du Triomphe, pour n'en citer que quelques-unes. Un aspect à souligner de LNT est l'absence d'éléments appartenant à la « culture savante ». En effet, nous ne trouvons que la référence à un film (La môme) et à deux chanteurs (Édith Piaf et Grand Corps Malade). Le reste des expressions artistiques (peinture, sculpture, littérature) est à peine représenté dans ce manuel.

Les sections culturelles sont riches en EIRC. Chacune de ces leçons contient en moyenne 4 images dans lesquelles apparaissent des personnalités françaises, des symboles de la France et des situations de la vie quotidienne. Au début du manuel, certaines personnalités françaises sont évoquées : Édith Piaf, Zinédine Zidane et Laure Manaudou. À mesure que les leçons progressent, les éléments culturels de la France deviennent la règle. Par exemple, dans la leçon 6 , les images sont consacrées au thème de l'emploi en France; dans la leçon 8, il y a des symboles tels que la Citroën 2CV, le fromage, le tour de France ; et dans la leçon 9, les images sont consacrées au pain.

En ce qui concerne le multiculturalisme, les images sont peu représentatives des autres cultures et nationalités : un pont à Montréal, le Tower Bridge à Londres, le parc 
Güell à Barcelone. Il est à noter que la première leçon de culture/civilisation est la seule consacrée spécifiquement à la francophonie. Nous y trouvons des photographies d'individus représentant différents pays où le français est parlé, mais elles sont des images d'archives n'exposant que de visages. Dans cette optique, la francophonie n'est pas un sujet très développé dans ce manuel.

Il s'avère donc que les éléments culturels de la France sont beaucoup plus représentés que ceux des autres pays francophones. Aucune personnalité appartenant à la francophonie n'est évoquée. Enfin, les EIRC correspondent le plus souvent à des monuments et à des lieux; il n'y a pas de références à des institutions ni à des expressions culturelles populaires.

\section{Alter Ego Plus (AEP)}

Ce manuel adopte également une structure systématique des unités : 3 leçons de 2 doubles pages par unité ou dossier. Elles commencent régulièrement par la mise en œuvre d'une situation communicationnelle. La section consacrée à la culture/civilisation s'intitule «Carnet de voyages » et concerne une double page en couleur riche en supports graphiques. Il est important de mentionner qu'il n'y a pas beaucoup d'exercices d'écriture à l'intérieur de chaque leçon. Ceux-ci sont rassemblés dans une section à la fin de l'unité. Cela permet d'obtenir une composition visuelle pleine d'images et d'activités actionnelles.

Tout comme $L N T$, les pages d'ouverture d'unité dans AEP présentent une grande image. II faut pourtant souligner que les éléments représentés dans ces pages n'ont pas de rapport immédiat avec la culture cible, car il s'agit le plus souvent d'images d'archives : un banc de poissons, une horloge, etc. Toutefois, à l'intérieur de chaque unité, la quantité d'images est supérieure à celle de $L N T$ : jusqu'à dix images par page dans les leçons générales. Dans le Tableau 2, nous exposons les résultats des images contenues dans AEP. En moyenne, il y a 2,15 images par page. Ce nombre est visiblement supérieur à celui rapporté dans $L N T$ (1,38 images par page). La distribution des catégories est aussi bien différente dans les deux manuels. Dans $L N T$, il y a des intervalles de 10 $\%$ entre les catégories les plus fréquentes et les moins fréquentes. En revanche, dans $A E P$, sauf les photographies authentiques, les autres catégories ont en moyenne une fréquence d'emploi presque similaire. Cela implique une utilisation plus équilibrée des différents types d'images à l'intérieur du manuel. 
Tableau 2: Nombre d'images retrouvées dans AEP

\begin{tabular}{|c|c|c|c|}
\hline \multicolumn{2}{|c|}{ Photographies } & \multicolumn{2}{c|}{ Illustrations } \\
\hline Authentiques & Adaptées & Authentiques & Créées \\
\hline $205(39 \%)$ & $120(23 \%)$ & $91(17 \%)$ & $109(21 \%)$ \\
\hline
\end{tabular}

En général, les photographies d'AEP établissent un rapport plus évident avec des éléments culturels. Si les images de $L N T$ n'étaient vraiment pas emblématiques de la culture cible - même pour les photographies authentiques - celles d'AEP font référence à des situations spécifiques. Par exemple, dans la leçon consacrée à Pontoise, I'Oise et les remparts y sont présentés, ainsi que la cathédrale de Saint-Maclou et le théâtre de l'Apostrophe. Ces EIRC donnent l'impression de voir la ville et de parcourir différents endroits.

Par ailleurs, bien que les illustrations soient la ressource la moins utilisée dans $A E P$, elles introduisent également des EIRC clés. D'une part, la manière de dessiner les personnages humains correspond au style conventionnel des manuels (que l'on trouve également dans $L N T$ ) : des figures peu ou prou stylisées à des traits caucasiens. ${ }^{3} D^{\prime}$ une autre part, il faut souligner que certaines illustrations authentiques viennent accompagnées de la source d'où elles ont été extraites. II s'agit d'une caractéristique de $L N T$ qui contribue certainement à créer un lien entre les EIRC présentés dans le manuel et les éléments culturels de la vie réelle.

En ce qui concerne la section culturelle d'AEP, les premières leçons comportent des photographies authentiques renvoyant à des EIRC de la France. Il s'agit notamment de symboles, de personnalités et de bâtiments de la France. Il faut souligner que certains EIRC ne sont pas facilement identifiables si l'on n'a pas une connaissance préalable de cette culture. Par exemple, nous trouvons des constructions assez modernes, telles que la Cité des sciences et de l'industrie, la façade du Musée du quai Branly ou de la Fondation Cartier. Les sujets des dernières leçons tournent autour des situations de la vie quotidienne, de l'histoire des monuments et de quelques expressions artistiques en France. En général, les images sont moins stéréotypées que dans LNT mais une explication est souvent nécessaire pour les situer dans leur contexte.

À la différence du manuel précédent, $A E P$ consacre toute une unité à la francophonie. Des images du Canada permettent d'introduire le vocabulaire des saisons et des

3 II reste à déterminer si ce style de représentation des individus répond à une certaine économie dans le dessin ou bien à une position culturelle sous-jacente. II s'agit en tout cas d'un recours stéréotypé très fréquent dans les manuels consultés qu'il ne faut pas négliger. 
photographies sur lî̂le de La Réunion illustrent les activités en plein air. Bien que les leçons soient cohérentes avec les images, celles-ci ressemblent à des images de type carte postale. En effet, il n'y a pas de personnalités canadiennes et les images de La Réunion semblent trop génériques. En revanche, le troisième pays représenté, la Belgique, a des images plus variées sur la ville et sur des éléments culturels reconnaissables: un tableau de Magritte, les bandes dessinées, l'Atomium. En général, les thèmes grammaticaux et lexicaux tirent le meilleur avantage de ces sujets culturels. À la fin de cette unité, il y a une leçon complémentaire sur la francophonie. Les matériels culturels comprennent la chanson « Je reviendrais à Montréal » de Robert Charlebois et une interview avec le compositeur sénégalais Tété.

Nous constatons un travail iconographique assez soigné dans $A E P$. Les images servent non seulement de support aux sujets communicatifs, mais elles semblent avoir un objectif culturel. Bien que ce manuel utilise, en moyenne, moins les photographies authentiques que $L N T$, les EICR sont mieux contextualisés. Cela donne l'impression d'une immersion culturelle plus réaliste et permet de laisser un peu de côté les clichés graphiques, ressource presque incontournable des manuels.

\section{3. Édito (EDI)}

La première différence de ce manuel avec les précédents est le nombre d'unités. Tandis que $L N T$ et $A E P$ comptent 9 unités, EDI en possède 12. La structure des unités est régulière, et les différentes compétences sont clairement différenciées. La section consacrée à la culture/civilisation se trouve au milieu de l'unité et ne diffère pas visuellement des autres leçons. L'emploi des couleurs est assez mesuré, le fond des pages étant normalement blanc.

La page d'ouverture de chaque unité contient une illustration réalisée pour le livre, qui a pour objectif « d'entrer avec humour dans le thème », comme indiqué dans le mode d'emploi du manuel. D'après nos données, il s'agit du manuel qui utilise le plus d'images : 2,5 images par page en moyenne. Dans le Tableau 3, nous exposons la distribution des différentes catégories repérées. Iconographiquement, l'une des caractéristiques principales d'EDI est un emploi très limité des illustrations par rapport aux photographies. En ce qui concerne les photographies, la fréquence d'emploi est similaire entre les sous-classes authentiques et adaptées. Or, il faut souligner que si les photographies authentiques sont un moyen efficace pour montrer des EIRC, ce 
n'est pas le cas des photographies adaptées ou d'archives, car elles présentent des éléments iconographiques génériques. Dans cette optique, seule une photo sur deux dans $E D I$ peut être reconnue comme une situation culturelle authentique. C'est la sensation obtenue lors de l'utilisation du manuel : de nombreuses images servent uniquement à illustrer un exercice ou une leçon.

Tableau 3: Nombre d'images retrouvées dans EDI

\begin{tabular}{|c|c|c|c|}
\hline \multicolumn{2}{|c|}{ Photographies } & \multicolumn{2}{c|}{ Illustrations } \\
\hline Authentiques & Adaptées & Authentiques & Créées \\
\hline $215(40 \%)$ & $220(41 \%)$ & $45(8 \%)$ & $61(11 \%)$ \\
\hline
\end{tabular}

En ce qui concerne les illustrations, il faut souligner deux aspects fondamentaux. D'une part, de nombreuses illustrations authentiques concernent des statistiques et des enquêtes qui tentent de capturer graphiquement des informations sur la vie et la société françaises. Elles sont souvent accompagnées de la source d'où elles ont été extraites. Comme nous l'avons indiqué, cette stratégie crée l'impression que le document reflète des informations authentiques de la culture cible. D'autre part, bien que les illustrations créées pour le manuel soient peu nombreuses, il y a une perspective multiculturelle très marquée surtout en ce qui concerne la représentation des personnages humains. En effet, il existe une grande diversité ethnique et physique des individus dessinés dans ce manuel : des variations dans la couleur de la peu, dans les traits et dans la physionomie. Ce type de représentation n'est pas attestée dans $L N T$ et n'apparaît que dans la leçon initiale d'AEP « Les uns, les autres ». En ce sens, $E D /$ présente une approche multiculturelle plus évidente que le reste des manuels consultés. Cela est particulièrement perçu dans la première moitié du manuel.

Une autre caractéristique d'EDI (qui d'ailleurs est rare dans $L N T$ et peu fréquente dans $A E P$ ) est la représentation des institutions, des événements et des personnalités liées à la culture francophone. Nous trouvons des personnalités francophones qui ne sont pas très connues sur la scène internationale, par exemple Youssu N'Dour ou Charline Vanhoenacker. Cette stratégie contribue également à renforcer l'idée de multiculturalisme. Elle est surtout récurrente dans la première partie du manuel, où le sujet de la francophonie et des différences culturelles est constamment traité. Dans la seconde partie, les thèmes tournent plutôt autour de la France. Du point de vue du contenu, les images se correspondent clairement avec les sujets communicatifs.

En ce qui concerne la section consacrée à la culture/civilisation, elle peut être divisée en deux sous-sections. La première correspond à la leçon intitulée « Civilisation » qui 
présente des éléments culturels de la vie quotidienne. La seconde, à la fin de chaque unité, correspond à la leçon « Détente », où différentes activités ludiques sont proposées. Les deux leçons sont riches en EIRC et adoptent une perspective multiculturelle dans la première partie du manuel.

En général, ce manuel propose une grande variété d'éléments graphiques multiculturels. Les EIRC contenus dans la page d'ouverture préconisent, d'une certaine manière, cette posture multiculturelle. En effet, des différences ethniques, physionomiques et humoristiques coexistent souvent dans ces dessins. C'est une caractéristique constante dans plusieurs leçons du manuel.

\section{Discussion et conclusion}

Dans cette étude, nous avons analysé les éléments culturels de trois manuels de FLE publiés à quelques années d'intervalle. Nous avons signalé des similitudes et des différences importantes dans la reproduction de ces éléments. Dans le graphique suivant (Figure 5), nous exposons une comparaison des emplois des EIRC retrouvés lors de notre analyse. Ces données révèlent d'abord que les photographies sont plus utilisées que les illustrations dans les manuels les plus récents. Cela pourrait confirmer une tendance généralisée à utiliser des images pour contextualiser la langue. Dans le cas des illustrations, elles sont fréquemment utilisées pour présenter des informations sur le mode de vie et les habitudes de la culture cible, et elles sont parfois accompagnées de la source d'où elles ont été tirées. Toutes ces stratégies iconographiques vont dans le sens de créer l'impression qu'il s'agit de documents authentiques et représentatifs de la culture cible.

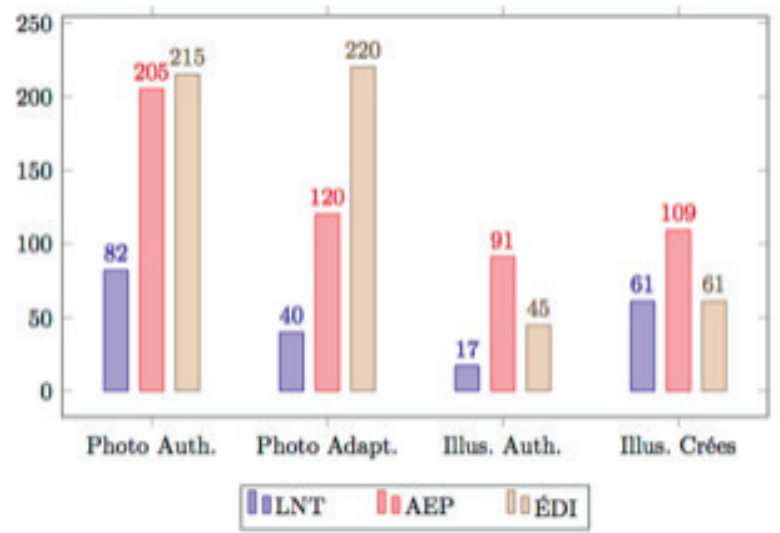

Figure 5: Comparaison des EIRC dans les trois manuels, d'après nos résultats 
En ce qui concerne le contenu culturel présenté dans les EIRC, les trois manuels accordent une place importante à la culture de la France. Les images décrivent très souvent des situations de la vie quotidienne de ce pays et les symboles évoqués sont principalement français. Cette position aussi bien théorique que méthodologique peut trouver son explication dans le concept de « français standard » proposé par Klinkenberg (1999), c'est-à-dire une langue (et également une culture) dans laquelle les membres d'une communauté se reconnaissent et peuvent être reconnus. En effet, les trois manuels présentent, de manière presque incontournable, des aspects iconographiques de la culture française dès leurs premières leçons. Suivant Agar (1994) et Block (2007), le contact entre la culture de l'apprenant et la culture cible produit une certaine déstabilisation. L'idée de recourir à certains stéréotypes iconographiques peut donc aller dans le sens de faire moins dérangeant ce premier contact.

Cependant, dans les manuels les plus récents, il y a un vrai intérêt à présenter des EIRC appartenant à d'autres cultures. En effet, dans plusieurs leçons d'AEP et d'EDI, nous constatons une présence fréquente de personnalités et d'institutions francophones, une description iconographique des pays où le français est parlé plus minutieuse et des dessins qui comportent des caractéristiques relevant du multiculturalisme. En ce qui concerne les leçons consacrées à la francophonie, ces manuels intègrent également un contenu culturel très diversifié. Contrairement à $L N T$ où la francophonie avait une fonction illustrative, $A E P$ et $E D$ I présentent un travail de recherche iconographique plus élaboré à cet égard. Nous pouvons donc établir que, dans ces derniers manuels, il y a un plus grand intérêt à présenter le multiculturalisme comme une autre voie pour apprendre la langue. Dans cette approche, déjà avancée par Puren (2002), I'apprenant peut réfléchir sur sa culture et celle d'autrui et forger une pensée multiculturelle.

Il s'avère que résumer iconographiquement une culture en quelques pages est une tâche difficile, mais il semble que la proposition actuelle des manuels va dans cette direction. Un équilibre entre le contenu communicatif et les EIRC constitue un aspect clé dans leur élaboration. Plus le contenu graphique est soigné, plus le manuel peut constituer une véritable fenêtre à la langue et à la culture d'autrui. 
Évaluation : Évaluation anonyme par des pairs extérieurs.

Conflit d'intérêts : L'auteur n'a aucun conflit d'intérêts à déclarer.

Subvention : L'auteur n'a reçu aucun soutien financier pour ce travail.

Peer-review: Externally peer-reviewed.

Conflict of Interest: The author has no conflict of interest to declare.

Grant Support: The author declared that this study has received no financial support.

\section{Bibliographie}

Agar, M. (1994). The intercultural frame. International Journal of Intercultural Relations18(2), 221-237.

Alcaraz, M., Braud, C., Calvez, A., Cornuau, G., Jacob, A., Vidal, S., \& Pinson, C. (2016). Édito 1. Méthode de français. Didier.

Ali, S., Kazemian, B., \& Mahar, I. (2015). The Importance of Culture in Second and Foreign Language Learning. Dinamika Ilmu, 15(1) 1-10.

Auger, N. (2001). Les formes du stéréotype dans les manuels scolaires de français langue étrangère : quelques usages pour quelques stratégies. Marges Linguistiques1, 35-56.

Barbier, C. (2013). La francophonie dans l'enseignement du Français Langue Étrangère. Analyse des manuels de FLE "Latitudes » et des représentations des enseignants du Département de Langues Étrangères de l'Université de Sonora. Universidad de Sonora. Thèse doctorale.

Berringer, P., Grandmangin, M., \& Rebérioux, A. (1995). Éditer des méthodes (table ronde). Français dans le monde. Numéro spécial. Méthodes et méthodologies, 17, 12-20.

Block, D. (2007). The rise of identity in SLA research, post Firth and Wagner (1997). The Modern language journal, $91,863-876$.

Brito Meyer, R. M. (2013). Cultural, multicultural, intercultural: O português como segunda língua para estrangeiros. Matraga-Revista do Programa de Pós-graduação em Letras da UERJ, 20, 54-65.

Capelle, G., \& Menand, R. (2009). Le nouveau taxi ! 1: Méthode de français. Hachette.

Castillo, E. D., \& Pérez, A. (2016). La motivation d'apprenants de français dans une université mexicaine. Synergies Mexique, 6, 81-91.

Chi, H. (2007). Représentation de la France des étudiants taïwanais de français à la fin de leur formation. Synergies Chine, 2, 246-266.

Chiswick, B. R., \& Miller, P. W. (2005). Linguistic distance: A quantitative measure of the distance between English and other languages. Journal of Multilingual and Multicultural Development, 26(1), 1-11.

Conseil de l'Europe. (2001). Le Cadre européen commun de référence pour les langues - Apprendre, Enseigner, Évaluer (CECRL). Éditions Didier.

Galisson, R. (1991). De la langue à la culture par les mots. CLE International.

Girardet, J. (1995). Profession auteur. Le français dans le monde. Numéro spécial. Méthodes et méthodologies, 17, 25-33.

González Izquierdo, E. (2014). Quelle(s) culture(s) et littérature(s) enseigner en cours de français en contextes scolaires espagnols ? Synergies Espagne, 7, 145-159. 
Kizirian, V. M., Daill, E., Berthet, A., Hugot, C., \& Waendendries, M. (2012). Alter Ego + 1 : Livre de l'élève. Hachette. Klinkenberg, J.-M. (1999). Des langues romanes. De Boeck Duculot.

Moore, D. (2005). Les Représentations des langues et de leur apprentissage : Références, modèles, données et méthodes. Didier.

Nguyen, P. N. (2008). Stéréotypie et méthodes de FLE. Synergies Espagne, 1, 195-204.

Porcher, L. (1995). Le français langue étrangère. Hachette.

Puren, C. (2002). Perspectives actionnelles et perspectives culturelles en didactique des langues-cultures : vers une perspective co-actionnelle-co-culturelle. Langues modernes, 96(3), 55-72.

Ranchon, G. (2016). Une didactique de la langue, de la culture et du genre : Le manuel FLE, discours et réalisations. Université de Lyon. Thèse doctorale.

Richaudeau, F. (1986). Conception et production des manuels scolaires : Guide pratique. Presses de I'UNESCO.

Schapira, C. (1999). Les stéréotypes en français : proverbes et autres formules. Éditions Ophrys.

Silva, H. (2011). Langues et territoires : le statut du français en Amérique Latine. Cahier de la Chaire Senghor de la Francophonie, 3.

Vajta, K. (2012). Le manuel de FLE suédois, véhicule d'un discours sur la France, communauté imaginaire. In E. Ahlstedt, K. Benson, E. Bladh, I. Söhrman, \& U. Akerström (Eds.), Actes du XVIIle congrès des romanistes scandinaves (pp. 747-763). 
